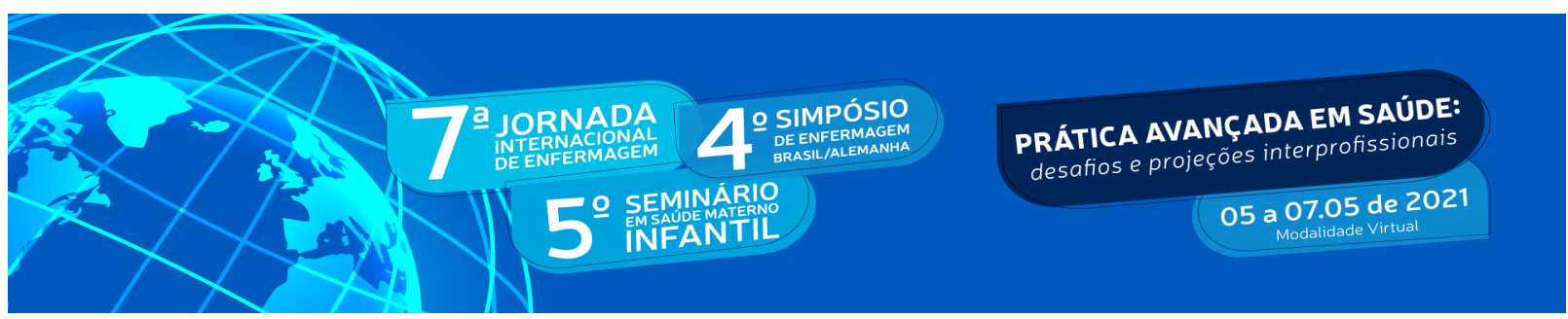

DOI: http://doi.org/10.48195/jie2021-054

\title{
O COMPROMISSO DA LIGA ACADÊMICA DE ATENÇÃO EM ONCOLOGIA COM OS PILARES DA UNIVERSIDADE ${ }^{1}$
}

\author{
Luisa de Assis Sobreira ${ }^{2}$; Sara Soares Ferreira da Silva ${ }^{2}$; Clara Beatriz Teixeira Lima \\ Cavalcante $^{2}$; Johnny Lima Brandão²; Karina Medeiros Santos²; Ana Cristina Silva \\ Pinto $^{3}$
}

\section{RESUMO}

Objetivo: relatar ações realizadas pela Liga acadêmica de Atenção em Oncologia (LAAONCO) sob a ótica dos pilares ensino-pesquisa-extensão. Método: estudo descritivo, do tipo relato de experiência, realizado a partir de registros da LAAONCO. Desenvolvimento: As ligas acadêmicas vêm ganhando destaque no cenário acadêmico devido a sua potencialidade, neste cenário se insere a LAAONCO, que vem desenvolvendo ações baseadas no pilar universitário. As ações do eixo Ensino consistem em palestras, cursos e rodas de conversa, utilizando-se de metodologias ativas de aprendizagem e construção do conhecimento de forma bilateral. No eixo Pesquisa são realizados treinamentos e produções científicas, com apoio à participação em eventos científicos. No eixo extensão, são desenvolvidas atividades voltadas ao público externo à Universidade sobre prevenção e autocuidado em oncologia. Conclusão: a LAAONCO tem propiciado espaços de aprendizado, produção científica e extensionista, com impacto à comunidade acadêmica e externa, contribuindo para cumprimento do papel da Universidade para a sociedade.

Palavras-chave: Ensino Superior; Estudantes de Ciências da Saúde; Sucesso Acadêmico; Oncologia; Educação em Enfermagem.

\begin{abstract}
Objective: to report actions carried out by the Academic League of Attention in Oncology (LAAONCO) from the perspective of the teaching-research-extension pillars. Method: descriptive study, of the type of experience report, carried out from LAAONCO records. Development: Academic leagues have been gaining prominence in the academic scenario due to their potential, in this scenario LAAONCO is inserted, which has been developing actions based on the university pillar. The actions of the Teaching axis consist of lectures, courses and conversation circles, using active methodologies for learning and building knowledge on a bilateral basis. In the Research axis, training and scientific productions are carried out, with support for participation in scientific events. In the extension axis, activities are developed aimed at the public outside the University on prevention and self-care in oncology. Conclusion: LAAONCO has provided spaces for learning, scientific production and extension, with an impact on the academic and external community, contributing to fulfill the role of the University for society.
\end{abstract}

Key Words: Education, Higher; Students, Health Occupations; Academic Success; Oncology; Education, Nursing.

Estudo original do tipo relato de experiência

${ }^{2}$ Estudante do Curso de Enfermagem. Universidade Federal do Estado do Rio de Janeiro (UNIRIO). E-mail: laaoncounirio@gmail.com

${ }^{3}$ Orientador. Doutora em Enfermagem. Universidade Federal do Estado do Rio de Janeiro (UNIRIO). E-mail: ana.pinto@unirio.br 


\section{INTRODUÇÃO}

As ligas acadêmicas (LA) são entidades estudantis, formadas por discentes de Instituições de Ensino Superior (IES), docentes e demais profissionais, sendo seu objetivo a expansão e disseminação do conhecimento sobre um determinada temática sem, contudo, propor a substituição do ensino da IES, mas o incremento e aprofundamento de conhecimentos a partir de demandas do corpo estudantil (DANTAS et al, 2017; HAMAMOTO FILHO, 2011).

Neste contexto, surge a Liga Acadêmica de Atenção à Oncologia (LAAONCO), no ano de 2019, a partir de iniciativa de grupo de discentes da Escola de Enfermagem Alfredo Pinto da Universidade Federal do Estado do Rio de Janeiro (UNIRIO), com o intuito de aprofundar os conhecimentos técnico-científicos acerca da oncologia, nos âmbitos de promoção da saúde, prevenção, detecção precoce, tratamento e cuidados paliativos, vindo de encontro à Política Nacional para a Prevenção e Controle do Câncer (BRASIL, 2013). Contudo, apesar de fundada por discentes de enfermagem, a liga foi instituída com a proposta da multidisciplinaridade devido a crescente visão, em âmbito mundial, da importância desta abordagem no contexto oncológico, uma vez que é irrealista a percepção de que uma profissão, somente, seja capaz de abranger toda a complexidade da oncologia (VALENTINI, 2014).

Além disto, a organização da LAAONCO se dá a partir dos pilares universitário Pesquisa-Ensino-Extensão, sendo tal conformação originada a partir da definição pela Constituição Federal Brasileira destes pilares para as Universidades, sendo estes articulados de maneira indissociável, definindo o papel universitário perante a sociedade (BRASIL, 1998). Neste sentido, o ensino entra com o intuito de propiciar formação de profissionais com habilidades e competências para atuar e contribuir dentro de sua área de atuação, já a pesquisa engloba a produção científica e, por fim, a extensão se dá de maneira a retornar os conhecimentos produzidos nas Universidades para a sociedade (SILVA et al, 2020).

Consequentemente, a LAAONCO atua de forma a agregar conhecimento técnico-científico aos discentes e público acadêmico; estimular a produção científica entre discentes de graduação e promover atividades extramuros universitários, acerca da oncologia em todas as etapas que perpassam seu processo saúde-doença.

\section{OBJETIVO}

Relatar ações realizadas pela Liga Acadêmica de Atenção em Oncologia sob ótica dos pilares Pesquisa-Ensino-Extensão. 


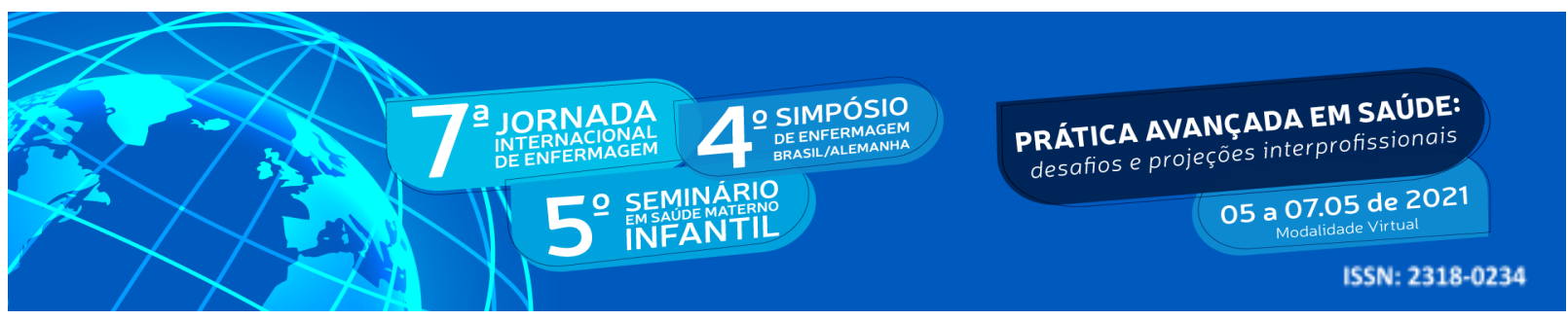

\section{METODOLOGIA}

O presente estudo trata-se de um estudo descritivo, do tipo relato de experiência, referente às ações da Liga Acadêmica de Atenção em Oncologia sob ótica dos pilares universitários Ensino-Pesquisa-Extensão.

Para reunião das informações apresentadas, foram consultados registros pertencentes à LAAONCO, sendo eles: atas de reuniões da liga, registros de eventos e atividades, banco de registros fotográficos, assim como, sucintos relatos pertinentes às ações da liga de cada membro diretor contemplado como autor do presente estudo. Foi adotado como recorte temporal o período compreendido desde a fundação da liga até o momento de escrita do presente estudo, isto é, agosto de 2019 a abril de 2021, totalizando um ano e oito meses.

Por se tratar estudo que se utiliza de informações de acesso público e de não envolver pesquisa com seres humanos, o presente estudo não foi submetido ao sistema $\mathrm{CEP} / \mathrm{CONEP}$ para apreciação, conforme resoluções nº510 de 2016 e n466 de 2012 do Conselho Nacional de Saúde (BRASIL, 2016; 2012).

\section{DESENVOLVIMENTO}

Um dos pilares essenciais para uma Liga Acadêmica é o Ensino, promovido através de estratégias e atividades capazes de abordar, discutir e enriquecer a temática proposta. $\mathrm{O}$ objetivo do ensino em uma LA é transmitir conteúdo teórico-prático de forma sistemática, complementando a aprendizagem e motivando a busca constante de novas informações (YANG et al., 2019).

$\mathrm{Na}$ LAAONCO, as atividades de ensino foram divididas em: (1) Atividades externas, isto é, envolvendo o público não vinculado à liga, integrando acadêmicos, profissionais e entusiastas pelo tema e; (2) Atividades internas, com membros da liga, com o objetivo de promover a sensibilização e aproximação com diversos temas voltados à oncologia. As atividades externas de Ensino foram realizadas a partir de diversas estratégias, como, palestras, simpósios, cursos e materiais midiáticos em redes sociais.

Quanto às palestras, a Liga convida profissionais especialistas nas temáticas, buscando proporcionar ricos debates e forte base teórica e profissional. No início da instituição da liga, 
eram realizados exclusivamente eventos presenciais, contudo, no decorrer de seu desenvolvimento, houve mudanças contextuais relevantes que impactaram o processo funcional, dentre elas, se destaca a pandemia por COVID-19 que requereu adaptações na forma de instituir dinâmicas educativas. Assim, foi empregado o uso de ferramentas digitais, sendo realizadas webinars com diversas temáticas em oncologia, com alcance de discentes de diversas regiões do Brasil.

Neste contexto, durante o ano de 2021, as palestras abordaram os temas: Assistência oncológica em tempos de pandemia; Oncogênese; Cuidados paliativos oncológicos; Câncer feminino e Práticas Integrativas e Complementares (PICS); Câncer de Mama e PICS; Uso do Canabidiol na dor oncológica; Oncohematologia; Novembro Azul e Saúde do Idoso e Linha de Cuidado no Câncer de Mama. Desta forma, é possível perceber o compromisso da LAAONCO quanto à oportunização do aprendizado da oncologia, apesar do período conturbado vivenciado pela sociedade em geral e, ainda, quanto à promoção deste ensino de maneira contextualizada integrada a diversos eixos temáticos, buscando abordar o câncer para além do olhar biomédico ainda muito forte no ensino tradicional.

Outra atividade de ensino realizada pela LAAONCO foi a elaboração, em parceria com a Liga Acadêmica de Pediatria Multidisciplinar (LAPEM), de um minicurso voltado à oncologia pediátrica que buscou enfatizar a importância da atuação de equipe multiprofissional na assistência à criança em processo oncológico e de seus familiares. $\mathrm{O}$ curso, vinculado à Pró-Reitoria de Extensão e Cultura da Universidade Federal do Estado do Rio de Janeiro (PROExC-UNIRIO), contou com 421 inscritos e continha material de apoio e testes para fixação do conteúdo, objetivando-se melhor aproveitamento do ensino promovido pela dinâmica.

Por fim, para além das atividades de ensino através de eventos, a LAAONCO também realiza postagens educativas lúdicas e transmissões ao vivo (lives) informativas nas redes sociais. Tal estratégia ganhou maior relevância no contexto pandêmico e se mostrou exitosa, visto que possibilita a síntese de temas através de uma linguagem acessível e estética que desperta o interesse do público-alvo. As postagens contêm informações sobre determinados tipos de câncer, com explanações sobre conceitos, causas, fatores de risco, prevenção e tratamento.

No mês de novembro, a Liga Acadêmica em apreço, em cooperação técnica com a Sociedade Brasileira de Enfermagem Oncológica (SBEO) e outras ligas de oncologia de demais IES, promoveu, em comemoração ao Dia Nacional de Combate ao Câncer, uma coletânea de postagens informativas durante a semana. Foram produzidos posts voltados aos 


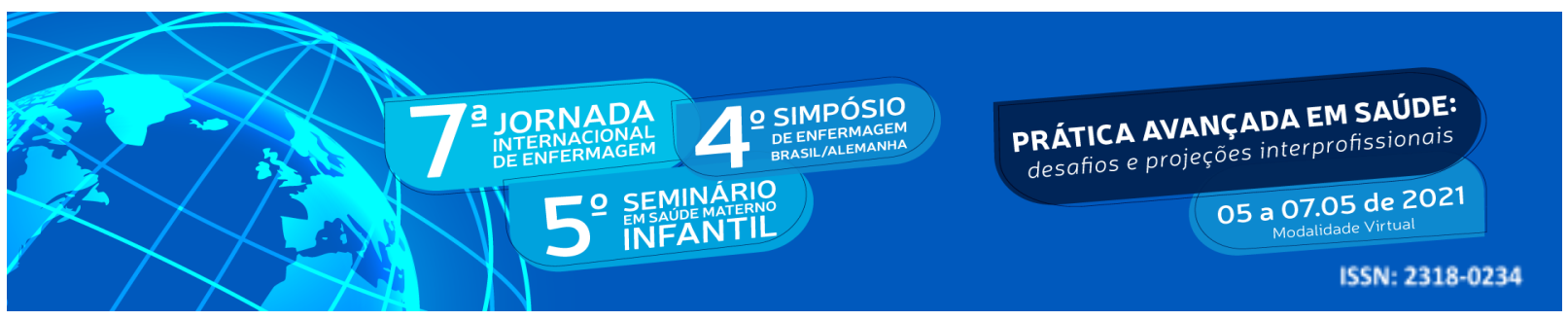

principais tipos de câncer e lives com palestrantes abordando os temas: Câncer de Mama; Câncer de Próstata e Alimentação Saudável e Câncer.

No que se refere às atividades internas de ensino, foram realizadas rodas de conversa com os membros da liga, quinzenalmente, acerca de temas em oncologia elencados previamente. Foi utilizada como estratégia a formação de grupos compostos por ligantes e diretores da liga, os quais eram responsáveis pela apresentação das temáticas e pela condução das dinâmicas. Através de dinâmicas lúdicas, com a oportunização do desenvolvimento do processo ensino-aprendizagem de forma bilateral, foi possível alcançar ampla abordagem dos diversos temas existentes na oncologia de forma otimizada e participativa, corroborando com o que traz Ramalho et al. (2012, p.72) que aponta o estímulo de ambientes de aquisição de conhecimento conduzidos pelos próprios acadêmicos como estímulo à "aquisição de conhecimento e experiência, desenvolvimento de raciocínio clínico-científico e promoção de trabalho em equipe".

Ademais, foi realizada a busca de cursos voltados à oncologia, gratuitos, em plataformas de cursos pertencentes a instituições renomadas, de forma a promover a oportunização do acesso ao conhecimento aos integrantes da liga. Assim, tais cursos eram divulgados aos membros periodicamente, sendo demonstrada grande aderência aos mesmos.

O câncer é um tema com diversas particularidades, a fim de incentivar a discussão das diversas temáticas que perpassam a oncologia, buscou utilizar-se de metodologias ativas de aprendizagem a fim de proporcionar o contato com temática tão importante, muitas das vezes abordada de forma escassa durante a graduação.

Outro pilar, de grande importância para as ações da LAAONCO, consiste na Pesquisa. Esta baseia-se na problematização e no questionamento, propiciando aos alunos um engajamento ativo nas atividades de investigação, contribuindo para o processo de aprendizagem (SOARES; CUNHA, 2017). Sendo a pesquisa um dos pilares para um ensino crítico e reflexivo, a liga acadêmica permite que, por meio da pesquisa, o aluno construa e desenvolva a investigação de fenômenos, atuando de forma complementar à aprendizagem e contribuindo para a disseminação dos saberes acadêmicos (YANG et al., 2019).

Além disso, de acordo com Araujo et al. (2019, p.140), "as ligas incentivam o desenvolvimento de trabalhos científicos, como forma de publicizar e compartilhar as 
experiências obtidas em campo, além de aprimorar as técnicas e métodos de produção científica". Por isso a LAAONCO, no âmbito da pesquisa, tem por objetivo, desenvolver pesquisa científica na área da oncologia, criando um espaço de oportunidade para que os alunos realizem atividades de pesquisa e participem de eventos científicos e de demais meios de divulgação científica.

Por meio da LAAONCO os alunos participam de um grupo de treinamento em pesquisa, contando atualmente com 10 alunos, dentre eles diretores e ligantes, e um professor orientador. O grupo realiza treinamentos sobre buscas em bases de dados e revisões da literatura, com produção de material audiovisual, e desenvolve estudos originais, de revisão e relatos de experiências. Atualmente, estão em fase de construção dois estudos, voltados ao processo de educação em saúde em oncologia e à atuação do enfermeiro no processo quimioterápico. Tais atividades buscam capacitar os alunos e promover maior contato com o campo da pesquisa.

Além disso, pensando na indissociabilidade entre pesquisa e ensino, a LAAONCO desenvolveu o "Estude com a LAAONCO", projeto que tem como objetivo divulgar artigos científicos atualizados na temática da oncologia, em sua página da rede social Instagram ${ }^{\circledR}$.

Fica evidente, através de suas produções científicas, a relevância da pesquisa na LAAONCO. Foram realizadas publicações em periódicos científicos nacionais e internacionais, voltadas à temática liga acadêmica e, ainda, ao processo de enfermagem voltado aos pacientes oncológicos. Ainda, foram realizadas apresentações de resumos em eventos científicos voltados às seguintes temáticas: (1) enfermagem e suas perspectivas enquanto profissão; (2) saúde da família e comunidade e (3) Sistema Único de Saúde brasileiro.

Dessa forma, a LAAONCO demonstra participar da produção e divulgação do conhecimento científico, propondo-se a fomentar a pesquisa na graduação, contribuindo para o desenvolvimento do aluno, da liga e da área de conhecimento.

A Extensão Universitária nas Instituições de Ensino Superior (IES) públicas se configura como uma forma de retribuir e retornar o investimento do setor público para a sociedade, estabelecendo ou reforçando o vínculo entre IES - que representam o âmbito acadêmico-prático - com a comunidade (KOGLIN; KOGLIN, 2019). No Brasil, ela foi baseada e desenvolvida inspirando-se em três distintos modelos universitários internacionais, que se moldam sobre os objetivos de disseminar conhecimentos, prestar serviços e tornar as IES acessíveis. (DUTRA; CASTIONI, 2017). 


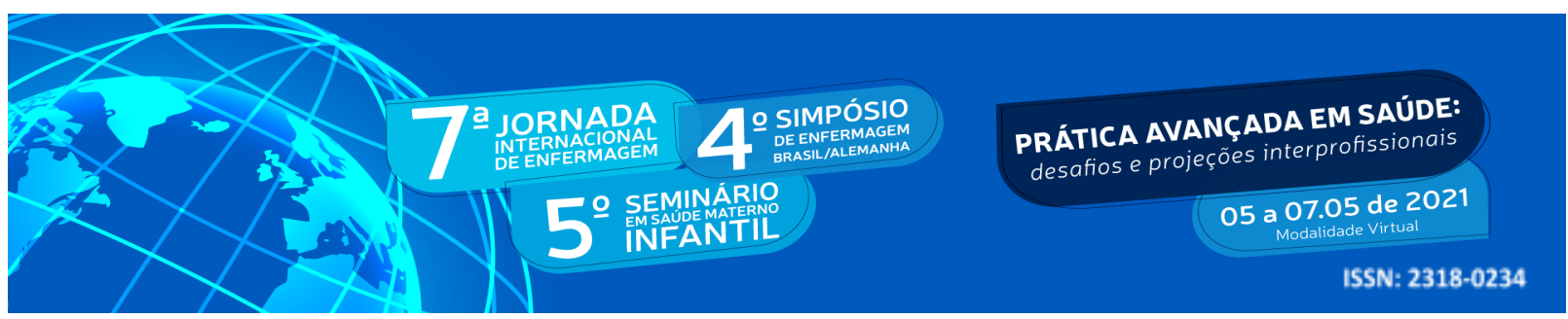

Sob essa perspectiva, a LAAONCO organiza a Tenda do Câncer de Mama, uma oficina sobre a temática do câncer de mama realizada, geralmente, em um hospital universitário. Objetiva-se com o evento, principalmente, a aprendizagem para usuários da Instituição em questão e a discussão sobre o tema e suas implicações. Ao longo da oficina, são abordados pontos sociais, clínicos, estatísticos, entre outros, como o exame clínico, o autoconhecimento das mamas, a rota do paciente oncológico ao longo da Rede de Atenção à Saúde (RAS) e a epidemiologia do câncer de mama, por exemplo.

Além disso, no atual momento de pandemia e entendendo que as Tecnologias da Informação e Comunicação (TIC) podem colaborar para o processo de educação em saúde (FRANÇA; RABELLO; MAGNAGO, 2019), a LAAONCO desenvolveu estratégias midiáticas a fim de disseminar informações e promover o conhecimento sobre oncologia ao público leigo. Dentre as estratégias desenvolvidas, encontram-se: 1) Elaboração de materiais educativos na rede social Instagram ${ }^{\circledR}$, em formato "carrossel”, com conteúdo informacional sobre temas específicos. Tal estratégia tem se mostrado exitosa, sendo os conteúdos com maior adesão do público: "COVID-19: Vacinação de Pacientes Oncológicos" e "Direitos Sociais da Pessoa com Câncer"; 2) Vídeos e oficinas, como "Oficina de Turbante" e "Fake News sobre o Câncer" e; 3) a estratégia "LAAONCO com você", que busca trazer receitas culinárias voltadas à indivíduos no contexto oncológico, de forma a promover alívio de sintomas e propiciar o conforto desta população.

Reiterando o compromisso com a educação em saúde, a liga continua desenvolvendo estratégias e pensando em novas formas de alcançar seus diversos públicos, de modo que já existem novos planejamentos dentro do campo da extensão. Dentre eles, encontram-se projetos capazes de proporcionar ou, até mesmo, exercitar aprendizados em Oncologia, auxiliando no aprofundamento teórico-prático dos participantes, além de novas atividades de extensão para o período pós-pandêmico.

Ademais, a liga tem como metas futuras o fortalecimento de maior aproximação com o campo assistencial, fortalecendo a integração ensino-serviço, através de parcerias já estabelecidas com instituições e associações em oncologia. 


\section{CONCLUSÃO}

As ações realizadas pela LAAONCO, relatadas no presente estudo, revelam seu papel enquanto entidade capaz de proporcionar aos acadêmicos, de diversas áreas do campo das ciências da saúde, amplas oportunidades de aprendizado sobre a oncologia, vindo de encontro às expectativas criadas desde sua fundação, além de alcançar êxito no âmbito extensionista e da pesquisa científica.

Desta forma, é possível identificar a importância da promoção de espaços de criação aos discentes durante a graduação, sendo alcançados, a partir destes, resultados com impactos à comunidade acadêmica e externa, contribuindo para o cumprimento do papel universitário perante a sociedade.

\section{REFERÊNCIAS}

ARAUJO, C. R. C. e.; LOPES R. E.; DIAS M. S. de A., XIMENES NETO F. R., FARIAS Q. L. T., CAVALCANTE A. S. P. et al. Contribuição das Ligas Acadêmicas para Formação em Enfermagem. Rev Enfermagem em Foco, [S.1.], v. 10, n. 6, p.137-142., 2019. Disponível em:

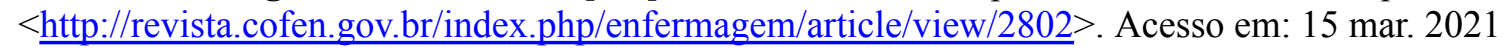

BRASIL. Ministério da Saúde. Conselho Nacional de Saúde. Resolução n ${ }^{\circ} 466$, de 12 de dezembro de 2012. Dispõe sobre as diretrizes e normas regulamentadoras de pesquisas envolvendo seres humanos. Brasília: Ministério da Saúde, 2012. Disponível em: $<\mathrm{http} / /$ bvsms.saude.gov.br/bvs/saudelegis/cns/2013/res0466_12_12_2012.html $>$. Acesso em: 12 mar. 2021.

BRASIL. Ministério da Saúde. Conselho Nacional de Saúde. Resolução no 510, de 07 de abril de 2016. Dispõe sobre as normas aplicáveis a pesquisas em Ciências Humanas e Sociais. Brasília: Ministério da Saúde, 2016.20 Disponível $<$ http://bvsms.saude.gov.br/bvs/saudelegis/cns/2016/res0510_07_04_2016.html >. Acesso em: 12 mar. 2021.

BRASIL. Ministério da Saúde. Portaria $\mathrm{n}^{\circ}$ 874, de 16 de maio de 2013. Institui a Política Nacional para a Prevenção e Controle do Câncer na Rede de Atenção à Saúde das Pessoas com Doenças Crônicas no âmbito do Sistema Único de Saúde (SUS). Brasília: Ministério da Saúde, 2013. Disponível em: <http://bvsms.saude.gov.br/bvs/saudelegis/gm/2013/prt0874_16_05_2013.html>. Acesso em: 12 mar. 2021.

DANTAS A.C.O, SANTOS M.A, GOIS M.B.C. et al. Importância da liga acadêmica para a formação profissional: aprendendo a trabalhar em equipe. Congresso Internacional de Enfermagem. v. 1 n. 1, 2017. Disponível em: $<$ https://eventos.set.edu.br/cie/article/view/6154/2429>. Acesso em 15 de mar. 2021.

DUTRA, N. L.; CASTIONI, R. A reforma de Córdoba e os projetos de universidade na América Latina - a proposta de Anísio Teixeira para o Brasil em que se assemelham? Integración y Conocimiento, [S.1.] v. 2, n. 7, p. 52-73, 2017. Disponível em:

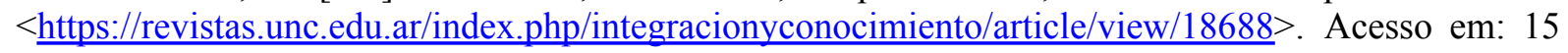
mar. 2021 


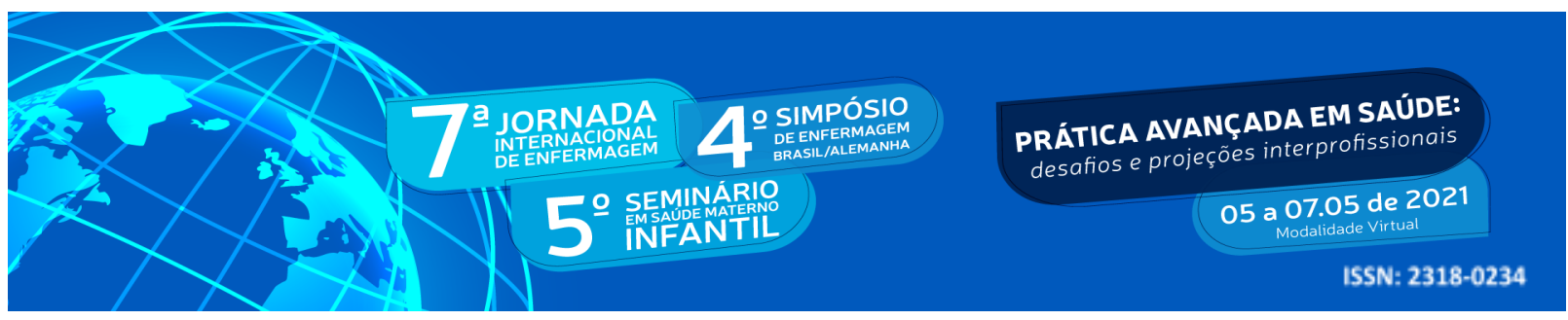

FRANÇA, T.; RABELLO, E. T.; MAGNAGO, C.. As mídias e as plataformas digitais no campo da Educação Permanente em Saúde: debates e propostas. Saúde em Debate [online]. [S.1.] v. 43, n. spe1, 106-115. Disponível em: $<$ https://www.scielo.br/scielo.php?script=sci_arttext\&pid=S0103-11042019000500106\&tlng=pt $>$. Acesso em: 11 mar. 2021.

HAMAMOTO FILHO P. T.; Ligas Acadêmicas: Motivações e Críticas a Propósito de um Repensar Necessário. Revista brasileira de educação médica. [S.1] 35(4): 535-543, 2011. Disponível em: $<$ https://www.scielo.br/scielo.php?script=sci_arttext\&pid=S0100-55022011000400013 $>$. Acesso em: 11 mar. 2021.

KOGLIN, T.; KOGLIN, J. C. A importância da extensão nas universidades brasileiras e a transição do reconhecimento ao descaso. Revista Brasileira de Extensão Universitária; [S.1.] v. 10, n. 2, p. 71-78, 7 jun. $72019 . \quad$ Disponível

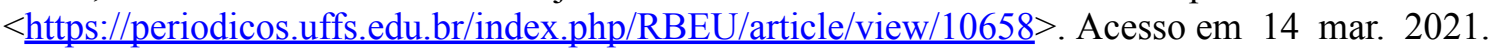

RAMALHO, A. S. et al. Anesthesiology teaching during undergraduation through an academic league: what is the impact in students' learning?. Rev. Bras. Anestesiol., Campinas , v. 62, n. 1, p. 68-73, fev. 2012 Disponível em: $<$ https://www.scielo.br/scielo.php?script=sci_arttext\&pid=S0034-70942012000100009\&lng=pt\&nrm= iso\&tlng=en>. Acesso em 14 mar. 2021.

SOARES, S. R.; CUNHA, M. I. Qualidade do ensino de graduação: concepções de docentes pesquisadores. Rev Avaliação (Campinas), Sorocaba, v. 22, n. 2, p. 316-331, ago. 2017.

Disponível em: $<$ https://www.scielo.br/scielo.php?script=sci_arttext\&pid=S1414-40772017000200316\&lng=pt\&tlng= pt>. Acesso em 15 mar. 2021

SILVA, S. S. F. da; CAVALCANTE , C. B. T. L. .; ANIZIO , M. de S. .; NUNES , B. L. R. .; PINTO, A. C. S. .; PAULA, D. G. de. Profile and production of academic health science leagues in Brazil: an integrative review. Research, Society and Development, [S. 1.], v. 9, n. 9, p. 743997775, 2020. Disponível em: https://rsdjournal.org/index.php/rsd/article/view/7775. Acesso em: 16 mar. 2021.

VALENTINI V. et al. Still a long way to go to achieve multidisciplinarity for the benefit of patients: commentary on the ESMO position paper. Annals of Oncology 25(1): 9-15, 2014. Disponível em: $<$ https://www.annalsofoncology.org/article/S0923-7534(19)35117-8/abstract\#secst0025>. Acesso em 16 mar. 2021

YANG, G. Y.-H. et al. Liga de Anatomia Aplicada (LAA): as Múltiplas Perspectivas sobre Participar de uma Liga Acadêmica. Rev. bras. educ. med., Brasília , v. 43, n. 1, p. 80-86, Mar. 2019. Disponível em: $<$ https://www.scielo.br/scielo.php?script=sci_arttext\&pid=S0100-55022019000100080\&tlng=pt $>$. Acesso em 14 Mar. 2021. 